\title{
Birefringence measurement of polymer photonic crystal fibers infiltrated with liquid crystals by depolarization of light effect
}

\author{
Daniel Budaszewski, ${ }^{* 1}$ Andrzej W. Domański, ${ }^{1}$ \\ ${ }^{I}$ Faculty of Physics, Warsaw University of Technology, Koszykowa 75, 00-662 Warszawa,
}

Received December 07, 2014; accepted December 29, 2024; published December 31, 2014

\begin{abstract}
In the paper we estimate group birefringence of polymer photonic crystal fiber partially infiltrated with a nematic liquid crystal $5 \mathrm{CB}$. To calculate group birefringence we use a novel method proposed by our group based on depolarization phenomena of partially coherent light that takes place in anisotropic media such as liquid crystals and birefringent optical fibers. Taking into consideration the spectral parameters of a light source and degree of polarization at the output of the fiber we calculated effective birefringence of the fiber partially infiltrated with a liquid crystal. The results for empty polymer optical fiber were compared with the data provided by the manufacturer to confirm the feasibility of the applied method.
\end{abstract}

Nowadays there has been growing interest in photonic liquid crystal fibers (PLCFs) and their applications in different areas of technology [1-4]. PLCFs combine the properties of a photonic crystal structure with electrooptical properties of liquid crystals (LCs). Generally, PLCFs are made of silica glass, but recently other materials have been used to produce optical fibers, i.e. polymers, which lead to create polymer optical fibers (POFs). The next step in fiber development is creation of microstructured polymer optical fibers (mPOFs) [5]. Unlike classical POFs, the mPOFs structure consists of microscopic air channels along the length of the fiber. The first microstructured polymer optical fiber made of polymethyl methacrylate PMMA was reported by Eijkelenborg et al. [6]. This material is most commonly used in manufacturing mPOFs, mainly due to their optical and mechanical properties. Nowadays, mPOFs are also manufactured from polycarbonate (PC) [7-8], ZEONEX [9], and TOPAS [10] polymers. One of the undoubted advantages of mPOFs in comparison to silica-glass optical fibers is their easier fabrication process by extrusion or drilling at low temperatures. However, high attenuation and sensitivity for damage can be considered as a disadvantage of mPOFs.

The vast majority of research studies have been devoted to the infiltration of a PLCF microstructure with nematic LCs, but recently also PLCFs infiltrated with smectic C* LCs have been reported [11].

In our research we have focused on birefringent mPOFs that can maintain polarization of guided light. In this type of mPOFs it is possible to observe depolarization phenomena of partially coherent light. In general, partially coherent light during propagation through birefringent media becomes depolarized [12]. The phenomenon of nonscattering depolarization of partially coherent light guided through different media has been investigated in anisotropic crystals [13], low-birefringent and high-birefringent silica-glass optical fibers [14, 15], as well as in liquid crystals [16]. The depolarization of light is caused by a phase shift that results from different velocities on both components of linearly polarized light due to the birefringence of an optical anisotropic medium. It also depends on the spectral parameters of a light source guided through a birefringent medium. The depolarization effect can be modified by infiltrating air holes of microstructured optical fiber with anisotropic materials, i.e. LCs characterized by two refractive indices, ordinary and extraordinary.

Depending on the value of effective birefringence of the PLCF as well as the length of the PLCF's infiltrated part it is possible to obtain any state of polarization (SOP) as well as different values of the degree of polarization (DOP) of a guided light beam. The value of DOP can be expressed by the following equations, for Lorentzian and Gaussian light sources respectively:

$$
\begin{aligned}
& P_{L}=\sqrt{1-\left[1-\exp \left(-\frac{2 \Delta N_{e f f} l}{L_{L}}\right)\right] \sin ^{2}(2 \theta)} \\
& P_{G}=\sqrt{1-\left\{1-\exp \left[-2\left(\frac{\Delta N_{e f f} l}{L_{G}}\right)^{2}\right]\right\} \sin ^{2}(2 \theta)}
\end{aligned}
$$

where:

$\theta$ - azimuth of linearly polarized light versus birefringence axis of the optical fiber,

$L_{L}=\frac{\lambda^{2}}{\Delta \lambda} \cdot \frac{1}{\pi}$ - coherence length of Lorentzian light source,

$L_{G}=\frac{\lambda^{2}}{\Delta \lambda} \cdot \frac{2 \sqrt{\ln (2)}}{\pi}$ - coherence length of Gaussian light source.

If the azimuth of incidental linearly polarized light is 45 degrees, then equations (1) and (2) can be reduced to the following form:

$$
P_{L}=\sqrt{\exp \left(-\frac{2 \Delta N_{e f f} l}{L_{L}}\right)}
$$

*E-mail: danielb@if.pw.edu.pl 


$$
P_{G}=\sqrt{\exp \left[-2\left(\frac{\Delta N_{e f f} l}{L_{G}}\right)^{2}\right]}
$$

Based on our results from previous research [17] it is possible to calculate the bandwidth in the case of a pure Lorentzian or Gaussian shape of the light spectrum. Also the coherence length or effective birefringence of the medium can be calculated by our method provided DOP and other parameters are known. Amongst many methods of measuring birefringence of optical materials, mostly interferometric methods are used [18-20]. Proposed by Chlebus et. al. [21], the method called spectral-domain tandem interferometry employs a complex setup of two interferometers. Another method called the wavelength scanning method [22] utilizes two polarizers at both endings of the fiber under test and determines group birefringence from spectral separation of interference fringes. All mentioned methods can be characterized by very good precision. Our depolarization method of group bierefringence measurement can be considered as a good alternative for the mentioned methods mainly due to its simplicity and cost-effectiveness. The simplicity of the proposed method for empty fiber is comparable to the wavelength scanning method, but gives less accurate results. Nevertheless, for very high transmission losses like in mPOFs infiltrated with LC, the proposed method seems to be more advantageous.

To investigate the effective birefringence of mPOFs we have measured DOP of empty and partially infiltrated mPOFs made of PMMA (Kiriama, Australia). The front view of the investigated $\mathrm{mPOF}$ is shown in Fig.1.

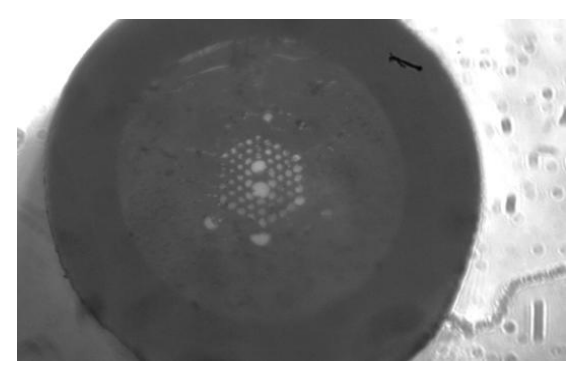

Fig. 1. A microstructure of the birefringent mPOF used in the experiment (Kiriama, Australia).

The group birefringence diagram provided by the producer of the investigated microstructured $\mathrm{mPOF}$ sample is shown in Fig. 2.

We have measured DOP of empty 168-cm-long mPOF using few laser diodes with different wavelengths and coherence lengths. The schematic setup is shown in Fig. 3.

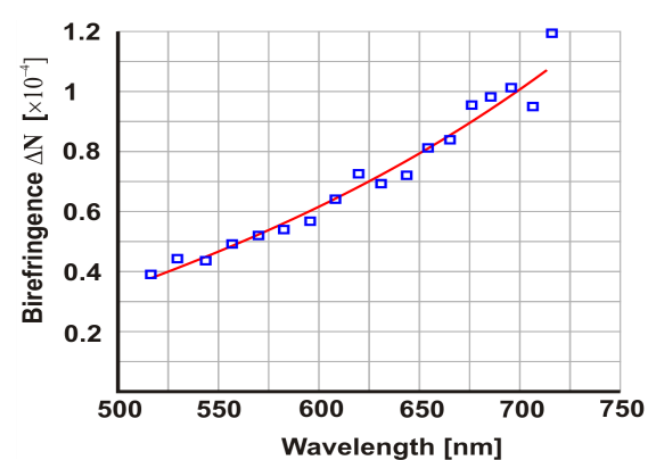

Fig. 2. Birefringence of the Kiriama microstructured mPOF provided by Kiriama.

What should be mentioned is that in this type of $\mathrm{mPOF}$ light propagation was based on a modified total internal reflection mechanism, caused by a higher refractive index of the core than the effective refractive index of the surrounding cladding.

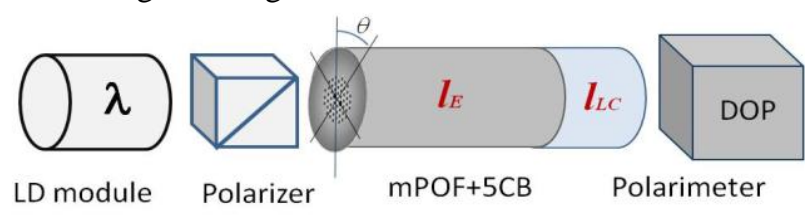

Fig. 3. Schematic setup used in the experiment. $l_{E}$ is the length of an empty $\mathrm{mPOF}$, and $l_{L C}$ is the length of an infiltrated part.

The light from a laser module was linearly polarized and coupled into mPOF under 45 degrees to excite both polarization components. The state and degree of polarization were measured at the output of the $\mathrm{mPOF}$ using a Stokes polarimeter THORLABS PAX 5004.

Afterwards, we calculated the effective birefringence of the mPOF using Eqs (5) and (6).

$$
\begin{aligned}
& \Delta N_{e f f}=\frac{L_{L}}{l} \cdot \ln \left(P_{L}\right) \\
& \Delta N_{e f f}=\frac{L_{G}}{l} \cdot \sqrt{\ln \left(P_{G}\right)}
\end{aligned}
$$

The results were compared with the data provided by the manufacturer. The parameters for these light sources were measured using an optical spectrum analyzer ANDO AQ 6315E. Table 1 presents light sources data and calculated effective birefringence.

\begin{tabular}{|c|r|r|r|r|}
\hline$\lambda[\mathrm{nm}]$ & $\begin{array}{c}\Delta \lambda \\
{[\mathrm{nm}]}\end{array}$ & DOP & $\Delta N_{\text {eff }}$ & $\begin{array}{c}\Delta N_{\text {eff }} \\
(\text { Kiriama })\end{array}$ \\
\hline 532.23 & 0.09 & 0.95 & $1.81 \times 10^{-5}$ & $4.17 \times 10^{-5}$ \\
\hline 674.34 & 0.16 & 0.90 & $12.6 \times 10^{-5}$ & $8.76 \times 10^{-5}$ \\
\hline 776.92 & 0.16 & 0.88 & $27.33 \times 10^{-5}$ & $14.19 \times 10^{-5}$ \\
\hline
\end{tabular}

Having compared the obtained results for an effective group birefringence, we can conclude that the proposed method gives comparable results, as for the order of magnitude, with the data provided by the manufacturer. 
The differences in the obtained values could be caused by fluctuations of the measured DOP values.

The experiment and calculations of an effective group birefringence were repeated for a 61-cm-long birefringent mPOF, partially infiltrated with a nematic LC 5CB on a distance of $1.5 \mathrm{~cm}$. The infiltration with $\mathrm{LC}$ was performed by capillary forces by dipping one end of the $\mathrm{mPOF}$ in a container with LC. Due to the fact that it was impossible to occlude smaller micro holes of the mPOF, all micro channels were infiltrated. It should be pointed out that in the filled part of mPOF the refractive index of the core was lower than the effective refractive index of the cladding with micro channels filled with LC. It means that for the infiltrated part of the mPOF, the light was guided by a photonic bandgap mechanism. Using a fiber optic spectrometer, we have checked which wavelengths can be guided in partially infiltrated mPOF under test. We have chosen three partially coherent light sources with a Lorentzian spectrum operating at the following wavelengths: $532 \mathrm{~nm}, 674 \mathrm{~nm}$ and $776 \mathrm{~nm}$. The measurement results for effective birefringence of empty $\mathrm{mPOF}$ and for partially infiltrated mPOF with LC are shown in Fig. 4.

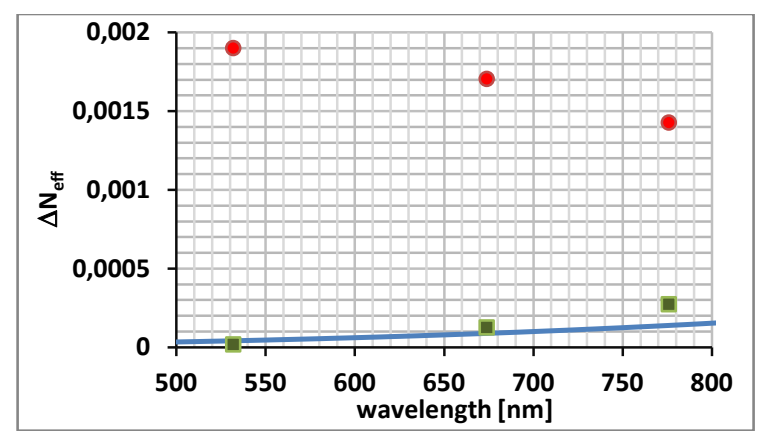

Fig. 4. Comparison of effective birefringence of empty mPOF given by Kiriama (blue curve) and experimental results (green squares), and partially infiltrated with LC (red dots).

According to our expectations, the effective birefringence of mPOF partially infiltrated with $5 \mathrm{CB}$ is higher than for an empty sample. The values of effective birefringence are shown in Table 2.

\begin{tabular}{|r|r|r|r|}
\multicolumn{1}{|c|}{ Table 2 } \\
\hline$\lambda[\mathrm{nm}]$ & \multicolumn{1}{|c|}{ DOP } & $\Delta n_{\text {eff }}\left(l_{E}+l_{L C}\right)$ & $\Delta n_{\text {eff }}\left(l_{L C}\right)$ \\
\hline 532 & 0.5 & $190.09 \times 10^{-5}$ & $9.05 \times 10^{-3}$ \\
\hline 674 & 0.32 & $170.5 \times 10^{-5}$ & $50.4 \times 10^{-3}$ \\
\hline 776 & 0,49 & $142.76 \times 10^{-5}$ & $51.11 \times 10^{-3}$ \\
\hline
\end{tabular}

Based on Eq. (5), we have made calculations for effective group birefringence of the filled part and found it to be two orders of magnitude higher than for empty mPOF.

The effective birefringence of infiltrated mPOF can be modified using an external electric field that can realign LC molecules inside mPOFs micro channels.

To conclude, we have investigated the group birefringence of mPOFs made of PMMA, using the measurement method based on depolarization phenomena. The applied method yields results comparable to those provided by the mPOFs manufacturer. Furthermore, the applied method is costeffective and less complex in application in comparison to other measurement methods used in fiber optic metrology. After partial infiltration of the investigated mPOF with nematic LC, we noticed an increase in effective birefringence, which was decreasing with the wavelength. A similar result was observed for silica glass PLCF infiltrated with the same nematic LC.

The research leading to this paper was supported by the National Science Center under the grant No. NCN 2011/01/B/ST7/05015.

\section{References}

[1] T.R. Woliński, S. Ertman, D. Budaszewski, M. Chychłowski, A. Czapla, R. Dąbrowski, A.W. Domański, P. Mergo, E. Nowinowski-Kruszelnicki, K.A. Rutkowska, M. Sierakowski, M. Tefelska, Phot. Lett. Poland 3, 20 (2011).

[2] S. Ertman, A.H. Rodríguez, M.M. Tefelska, M.S. Chychłowski, D. Pysz, R. Buczyński, E. Nowinowski-Kruszelnicki, R. Dąbrowski, T.R.Woliński, IEEE J. Lightwave Technol. 30, 1208 (2011).

[3] M.M. Tefelska, M.S. Chychłowski, T.R. Woliński, R. Dąbrowski, W. Rejmer, J. Wójcik, Acta Phys. Pol. A 118, 1259 (2010).

[4] Siarkowska, M. Jóźwik, S. Ertman, T.R. Woliński, V.G. Chigrinov, Opto-Electron Rev. 22, 3, 178, (2014).

[5] A. Argyros, J. Lightwave Technol. 27, 1571 (2009).

[6] M.A. Eijkelenborg, M.C.J. Large, A. Argyros, J. Zagari, S. Manos, N.A. Issa, I. Bassett, S. Fleming, R.C. McPhedran, C.M. de Sterke, N.A.P. Nicorovici, Opt. Expr. 9, 319 (2001).

[7] A. Stefani, K. Nielsen, H.K. Rasmussen, O. Bang, Opt. Commun. 285, 1825 (2012).

[8] C. Markos, A. Stefani, K. Nielsen, H.K. Rasmussen, W. Yuan, O. Bang, Opt. Expr. 21, 4758 (2013).

[9] J. Anthony, R. Leonhardt, A. Argyros, M.C.J. Large, J. Opt. Soc. America B 28(5), 1013 (2011).

[10] G. Emiliyanov, J.B. Jensen, O. Bang, P.E. Hoiby, L.H. Pedersen, E.M. Kjaer, L. Lindvold, Opt. Lett. 32, 460 (2007).

[11] D. Budaszewski, A.K. Srivastava, A.M.W. Tam, T.R. Woliński, V.G. Chigrinov, H-S. Kwok, Opt. Lett. 39, 16 (2014).

[12] W.K. Burns, J. Lightwave Technol. LT-1, 3, 475 (1983).

[13] A.W. Domański, Opto-Electron. Rev. 13, 171-176 (2005).

[14] J.I. Sakai, S. Machida, T. Kimura, IEEE J.Quantum Elect. OE-18, 488-495 (1982).

[15] A.W. Domański, M.A. Karpierz, A. Kujawski, T.R.Woliński, Proc. 12th Intern. Congress Laser 95, Springer-Verlag, Berlin, 684-687 (1996).

[16] A.W. Domański, D. Budaszewski, M. Sierakowski, T.R. Woliński, Opto-Electron. Rev. 14, 61-66 (2006).

[17] D. Budaszewski, A.W. Domański, T.R. Woliński, IEEE Instrumentation and measurement technology conference, IMTC 2007 (2007).

[18] N. Shibata, A. Nakazono, Y. Inoue, J. Lightwave Technol. 23, 3 (2005).

[19] F. Tang, X. Wang, Y. Zhang, W. Jing, Appl.Opt, 46, 19 (2007).

[20] P. Hlubina, T. Martynkien, W. Urbanczyk, Meas. Sci. Technol. 14, 784 (2003).

[21] R. Chlebus, P. Hlubina, D. Ciprian, Optics and Lasers in Eng. 47, 173 (2009).

[22] P. Hlubina, M. Szpulak, L. Knyblova, G. Statkiewicz, T. Martynkien, D. Ciprian, W. Urbanczyk, Meas. Sci. Technol. 17, 626 (2007). 